Published in final edited form as:

J Inherit Metab Dis. 2019 March ; 42(2): 325-332. doi:10.1002/jimd.12021.

\title{
Factor VIII and vWF deficiency in STT3A-CDG
}

\author{
Irene J. Chang ${ }^{1}$, Heather M. Byers ${ }^{2}$, Bobby G. Ng ${ }^{3}$, John Lawrence Merritt II ${ }^{1}$, Reid \\ Gilmore $^{4}$, Shiteshu Shrimal ${ }^{4}$, Wei Wei ${ }^{5,6}$, Yuan Zhang ${ }^{5,6}$, Amanda B. Blair ${ }^{7}$, Hudson H. \\ Freeze $^{3}$, Bin Zhang ${ }^{5,6}$, Christina Lam ${ }^{1}$ \\ ${ }^{1}$ Department of Pediatrics, Division of Medical Genetics, University of Washington, Seattle, \\ Washington \\ ${ }^{2}$ Department of Pediatrics, Division of Medical Genetics, Stanford University, Stanford, California \\ ${ }^{3}$ Human Genetics Program, Sanford Burnham Prebys Medical Discovery Institute, La Jolla, \\ California \\ ${ }^{4}$ Department of Biochemistry and Molecular Pharmacology, University of Massachusetts Medical \\ School, Worcester, Massachusetts \\ ${ }^{5}$ Genomic Medicine Institute, Cleveland Clinic, Cleveland, Ohio \\ ${ }^{6}$ Lerner Research Institute, Cleveland Clinic, Cleveland, Ohio \\ ${ }^{7}$ Department of Pediatrics, Division of Hematology-Oncology, University of Washington, Seattle, \\ Washington
}

\section{Abstract}

STT3A-CDG (OMIM\# 615596) is an autosomal recessive N-linked glycosylation disorder characterized by seizures, developmental delay, intellectual disability, and a type I carbohydrate deficient transferrin pattern. All previously reported cases $(n=6)$ have been attributed to a homozygous pathogenic missense variant c.1877C >T (p.Val626Ala) in $S T T 3 A$. We describe a patient with a novel homozygous likely pathogenic missense variant c.1079A $>\mathrm{C}$ (p.Tyr360Ser) who presents with chronically low Factor VIII (FVIII) and von Willebrand Factor (vWF) levels and activities in addition to the previously reported symptoms of developmental delay and seizures. VWF in our patient's plasma is present in a mildly hypoglycosylated form. FVIII antigen

\footnotetext{
Correspondence Christina Lam, MD MS MB.8.632 PO Box 5371 Seattle, WA 98145-5005 USA., ctlam2@uw.edu. AUTHOR CONTRIBUTIONS

Christina Lam conceived, planned, and wrote the manuscript, provided clinical care to the patient, and corresponded with collaborators and coordinated care and sample dispersion.

Irene J. Chang conceived, planned, and wrote the manuscript, and provided clinical care to the patient.

Heather M. Byers and J Lawrence Merritt II provided initial diagnosis and provided clinical care to the patient.

Bobby G. Ng initiated and coordinated patient testing amongst clinicians and collaborators. Hudson Freeze and Bobby G. Ng performed genetic testing and identification of the causative variant.

Reid Gilmore and Shiteshu Shrimal performed fibroblast glycosylation studies and western blotting.

Wei Wei, Yuan Zhang, and Bin Zhang performed VWF immunoblotting and FVIII secretion studies.

Amanda B. Blair provided clinical care to the patient.

All authors have reviewed, revised, and approved the manuscript as submitted.

CONFLICTS OF INTEREST

The authors have no conflicts of interest to report.

SUPPORTING INFORMATION

Additional supporting information may be found online in the Supporting Information section at the end of the article.
} 
levels were too low to quantify in our patient. Functional studies with $S T T 3 A^{-1-}$ HEK293 cells showed severely reduced FVIII antigen and activity levels in conditioned media $<10 \%$ expected, but normal intracellular levels. We also show decreased glycosylation of STT3A-specific acceptors in fibroblasts from our patient, providing a mechanistic explanation for how STT3A deficiency leads to a severe defect in FVIII secretion. Our results suggest that certain STT3A-dependent Nglycans are required for efficient FVIII secretion, and the decreased FVIII level in our patient is a combined effect of both severely impaired FVIII secretion and lower plasma VWF level. Our report expands both the genotype and phenotype of STT3A-CDG; demonstrating, as in most types of CDG, that there are multiple disease-causing variants in $S T T 3 A$.

\section{Keywords}

CDG; congenital disorder of glycosylation; developmental delay; low factor VIII activity; oligosaccharyltransferase; seizures; STT3A

\section{1| INTRODUCTION}

Congenital disorders of glycosylation (CDG) are a genetically and clinically heterogeneous group of diseases involving various defects in glycosylation pathways. One of the most ubiquitous pathways, $\mathrm{N}$-glycosylation, involves the covalent attachment of glycans to the side chain amide group of asparagine (Asn) residues within a consensus Asn-X-Ser/Thr (X $\neq$ Pro) acceptor site in the lumen of the endoplasmic reticulum (ER), trimming of terminal glucose residues, and further modification of the $\mathrm{N}$-glycan chain within the Golgi. ${ }^{1,2}$ The transfer of the glycan chain from the dolichol phosphate to the peptide chain is catalyzed by the oligosaccharyltransferase (OST) complex, a transmembrane enzyme complex in the ER. In humans, the highly conserved catalytic subunit of OST (STT3 proteins) is encoded by the $S T T 3 A$ and $S T T 3 B$ genes. STT3A mediates the co-translational glycosylation of nascent polypeptide chains, while STT3B mediates posttranslational glycosylation of any residual acceptor sites after translocation of the glycosylation motif. ${ }^{3}$

STT3A-CDG (OMIM\# 615596) is an N-linked glycosylation defect caused by pathogenic variants in the $S T T 3 A$ gene on chromosome 11q23.3, falling under the broader diagnostic umbrella of CDG. This disorder has been reported in two families with the same missense pathogenic variant, c.1877C $>\mathrm{T}$ (p. Val626Ala), which has been speculated to be a Pakistani founder variant. ${ }^{4,5}$ Although the phenotypic spectrum of STT3A-CDG has yet to be fully characterized given its recent discovery, the few patients described in the literature share common features of seizures, developmental delay, and intellectual disability. ${ }^{4,5}$ Variable features include microcephaly, self-injurious behavior, stereotypic movements, feeding problems, sleep disturbance, and episodic lethargy and hypothermia. ${ }^{4}$

Coagulation factors and inhibitors, including Factor VIII (FVIII) and von Willebrand Factor (vWF), are mostly N-linked glycoproteins. Patients with other types of CDG have a unique coagulation profile with significantly decreased levels of Factors IX and XI, Antithrombin III (ATIII), Protein C, and/or Protein S. ${ }^{6}$ However, clinical or subclinical coagulopathies have not been reported in patients with STT3A-CDG. Here, we describe a patient with a novel homozygous likely pathogenic missense variant c.1079A $>$ C (p.Tyr360Ser) in STT3A 
who presents with chronically low FVIII and vWF levels and activities, in addition to previously reported symptoms.

\section{2 | MATERIAL AND METHODS}

\subsection{Exome sequencing and analysis}

Exome sequencing was performed on the Illumina HiSeq platform in methods previously described. ${ }^{7}$ No other pathogenic or rare variants were identified in known glycosylationrelated genes, or in $F 8, V W F$, and SERPINC1 that may account for the patient's coagulopathy phenotype.

\section{2| Biochemical analysis}

Carbohydrate deficient transferrin isoform (CDT) analysis was performed using affinity chromatography and mass spectrometry. ${ }^{8}$ All other biochemical analyses were performed in a standard clinical lab.

\section{3| Fibroblast assay}

Primary skin fibroblasts from patients and controls were cultured as described previously. ${ }^{9}$ Cells were transfected with reporter plasmids for pSAP-DDKHis or sex hormone binding globulin (SHBG) as described previously. Glycoprotein substrates were pulse labeled with Tran35S label (Perkin Elmer) as described previously. ${ }^{5}$ As indicated, immunoprecipitated proteins were digested with endoglycosidase H (New England Biolabs). Dry gels were exposed to a phosphor screen (Fujifilm), scanned in Typhoon FLA 9000 and quantified using Image Quant TL software (GE scientific) to determine the percentage of pSAP and SHBG chains that have specific number of glycans.

\subsection{Peptide-N-glycosidase F (PNGase F) treatment of plasma and detection of vWF by immunoblotting}

Normal control plasma ( $2.25 \mu \mathrm{L}$ ) was digested with PNGase F (New England BioLabs, Ipswich, Massachusetts) in a $20 \mu \mathrm{L}$ reaction volume as described earlier. ${ }^{10}$ The reaction was stopped by the addition of $5 \mu \mathrm{L}$ of the $5 \times$ SDS sample buffer. Normal control plasma was diluted 1:20, and patient plasma was diluted 1:4 with the SDS sample buffer. Ten microliters of each sample were run in a 3-8\% NUPAGE gel with the Tris-acetate buffer (ThermoFisher, Waltham, Massachusetts), transferred to a nitrocellulose membrane and probed with a monoclonal anti-VWF antibody (ThermoFisher).

\subsection{FVIII secretion in STT3A and STT3B deficient cells}

The pMT2-FVIII plasmid, which contains the full-length FVIII cDNA, was transfected into wild-type (WT), STT3 $A^{-/-}$and $S T T 3 B^{-/-}$HEK293 cells as previously described. ${ }^{10}$ FVIII antigen levels in cell lysates and conditioned media were measured by an ELISA as previously described. ${ }^{10,11} \mathrm{FVIII}$ activity levels in conditioned media were measured using a chromogenic assay as previously described. ${ }^{10,11}$

All procedures followed were in accordance with the ethical standards of the responsible committee on human experimentation (institutional and national) and with the Helsinki 
Declaration of 1975, as revised in 2000. Informed consent was obtained from all patients for being included in the study. The study received exempt status by the Institutional Review Board of Seattle Children's Hospital. The patient's family gave written informed consent for this study. This article does not contain any studies with human or animal subjects performed by the any of the authors.

\section{3 | RESULTS}

\subsection{Clinical report}

The study received exempt status by the Institutional Review Board of Seattle Children's Hospital and the patient's family provided written informed consent for this study. The female patient was born at 40 weeks' gestation via Cesarean section for breech presentation to a consanguineous 33-year-old primigravid mother and a 35-year-old father. The pregnancy was uncomplicated and there were no maternal exposures. In the newborn period, the patient did not have good facial tracking and had noticeable left hypotropia associated with a marked limitation of elevation of the left eye. She also had bilateral ptosis. These abnormalities worsened over time and she developed large angle esotropia and strabismus with cortical visual impairment at 9 months of age. She had left ptosis with a diminished lid crease and lagophthalmos in downgaze and continued left hypotropia and poor visual attention. On dilated fundus exam, she had slightly dysmorphic-appearing disks, but no optic nerve hypoplasia, optic atrophy, or papilledema. She underwent eye alignment surgery at 3 years 11 months of age.

Her growth and development was reportedly normal until 3 months of age, when she was hospitalized for new onset generalized seizures. The patient initially presented with 5+ generalized seizures within the span of 1 day lasting 1 minute each; she was started on Keppra but continued to have up to six seizures lasting 3 minutes each per day while inpatient. Therefore, ACTH, Zonisamide, and Klonopin were added. EEG captured seizures and showed epileptic activity involving both hemispheres in a hypsarrhythmia background. The patient did not have any episodes of decreased consciousness or hypothermia; her axillary and rectal temperatures over 20 days of inpatient hospitalization ranged from 35.7 to $37.7^{\circ} \mathrm{C}$. At home, her seizures were fairly well controlled $(<2-3$ seizures per week lasting $<3$ minutes) on zonisamide, clobazam, and rufinamide until she started having $>3$ generalized breakthrough seizures a day at 2 years of age. At her maximum, she was having $>13$ seizures a day consisting of screaming, back-arching, and facial twitching with no identifiable triggers. At 3 years 11 months of age, she continues to have intractable epilepsy $(<3$ generalized seizures a week) with breakthrough cluster seizures on nearly a monthly basis. Her current AED regimen consists of Lacosamide $10 \mathrm{mg}$ twice daily $(1.75 \mathrm{mg} / \mathrm{kg} / \mathrm{day})$, Vigabatrin $750 \mathrm{mg}$ in the morning, $1000 \mathrm{mg}$ at nighttime $(153 \mathrm{mg} / \mathrm{kg} /$ day $)$, Clonazepam 0.5 $\mathrm{mg}$ at bedtime, and Diastat PRN. Family history was significant for parents being first cousins of Indian ancestry. On clinical evaluation at 3 months of age, she had inverted nipples, abnormal fat distribution, and esotropia suspicious for a congenital disorder of glycosylation (Figure 1). She had microcephaly (-2 SD), growth delay (height $-1.8 \mathrm{SD}$ and weight $-3.3 \mathrm{SD})$, and bilateral fifth toe clinodactyly. 
The patient had a 10-word vocabulary at age 2, which regressed to no speech at 3 years of age. Per parental report, the sudden decline in speech was precipitated by a long international flight. She was not ill during the flight but did suffer some jet lag and fatigue; upon arrival at their destination, she had stopped speaking and has never resumed any language abilities since then. She continued to have global developmental delay, truncal hypotonia, and could not yet walk, although she could sit with assistance and scoot. She had stereotypic hand-flapping and self-biting behaviors. She also had significant strabismus and did not track; visual evoked potential indicates significant cortical visual impairment. She had a total score of 31 (severe category, >26) on the Nijmegen Pediatric CDG Rating Scale for ages 2-11 years old based on current function, system specific involvement, and current clinical assessment. ${ }^{12}$

MRI brain at 1 year of age for seizures showed mild prominence of extra-axial spaces overlying the bilateral frontotemporal lobes. Head CT at 2 years of age for break-through and worsening seizures was normal. Echocardiogram at 1 year of age showed a small PDA and PFO. Renal ultrasound was normal. Polysomnogram at 2 years of age revealed mild obstructive sleep apnea with AHI of 5.3/hour (normal none/minimal <5/hour).

\subsection{Biochemistry}

CDT analysis at 1 year of age showed mono-oligo/di-oligo ratio of 0.55 (normal $<0.06$ ) and A-oligo/di-oligo ratio of 0.032 (normal $<0.011$ ), a pattern consistent with CDG type 1. Enzyme testing for phosphomannomutase and phosphomannose isomerase at Mayo Medical Laboratories were both normal. FVIII activity was chronically low at 6-10\% (normal range 50-150), but Factor V, IX, and XI activities were normal. Platelet count was $142-286 \mathrm{k} / \mathrm{mm}^{3}$ (normal range 150-450), vWF activity was at 35\% activity (normal range 50-200), and vWF antigen was $21 \%$ (normal range 35-241\%). vWF multimer analysis showed decreased concentrations of all multimers. ATIII activity was low at 53\% (normal range 75-125). APTT was high at 55-61 seconds (normal 25-35), PT and INR were normal. She did not have any clinical episodes of prolonged bleeding or easy bruising. Other biochemical investigational testing that were normal included urine organic acids, sulfites, creatine, and guanidinoacetate, purines and pyrimidines, argininosuccinic acid, plasma ammonia, acylcarnitine profile, and plasma and CSF amino acids. Uric acid was initially elevated at $3.2 \mathrm{mmol} / \mathrm{L}$ (normal range $0.5-2.2$ ), but normalized to 1.1 on repeat. Liver transaminases were slightly elevated (AST 51 IU/L (normal range 5-41), ALT 53 IU/L (normal range 645)).

\subsection{Molecular results}

SNP array performed for concern for copy number variants and autosomal recessive conditions given family history of consanguinity causing epilepsy and dysmorphic features showed no clinically significant deletions or duplications, but approximately $7.4 \%$ of the autosomal genome with loss of heterozygosity. On review of genomic coordinates (Genomic Oligoarray and SNP array evaluation tool v3.0), four candidate genes known to be pathogenic for CDGs were identified ( $A L G 1, A L G 11, S T T 3 A$, and COG6). Subsequently, a 34-gene panel for CDGs showed a single heterozygous variant of unknown significance p.Val432Leu in MOGS. A broader 65-gene CDG panel was negative. STT3A was not 
included in either panel. Research exome sequencing revealed a homozygous variant, c. 1079A >C (p.Tyr360-Ser), in STT3A (NM_152713.3), which was confirmed through clinical exome sequencing. This variant was predicted by in silico tools to be deleterious by SIFT, probably damaging by PolyPhen-2, disease causing by MutationTa-ster2, most likely to interfere with function by Align-GVGD, and deleterious by Provean. No additional pathogenic variants were found including in $F 8, V W F$, and SERPINC1.

\section{4 | Fibroblast assay}

Prosaposin (pSAP) is a well-established substrate of the STT3A complex ${ }^{3}$ that has five Nglycosylation sites. Pulse labeling experiments show that the major form of pSAP synthesized by HEK293 cells, control, and patient fibroblasts has five N-linked glycans (Figure 2, Panel A). However, there is an increased amount of pSAP chains that migrate more rapidly due to the absence of two glycans in patient cells as indicated in the bar graph. Quantification of the pSAP glycoforms revealed that the average number of glycans/pSAP chain decreased from 4.7 to 4.3 in the patients' cells compared to control fibroblasts or HEK293 cells (Figure 2, Panel A). For comparison, pSAP has on average two glycans per chain in a HEK293 derived STT3A null cell line, indicating that STT3A Y360S mutation is hypomorphic.. ${ }^{13}$ SHBG is a STT3B dependent substrate with two glycosylation sites, one of which shows partial occupancy. ${ }^{5}$ Glycosylation of SHBG in patient fibroblasts was intermediate between control fibroblasts and HEK293 cells (Figure 2, Panel B) indicating that $\mathrm{N}$-glycosylation by the STT3B complex is normal in the patients fibroblasts. As a reference, SHBG has 0.2 glycans per chains in a HEK293 derived STT3B null cell line. ${ }^{13}$ A western blot using anti-STT3A antisera did not show significant difference between control and patient sample ( $\pm 15 \%$, data not shown).

\subsection{Plasma vWF is hypoglycosylated}

We compared $\mathrm{vWF}$ in normal control and patient plasma by immunoblotting, which showed that patient $\mathrm{vWF}$ was present at a much lower level in plasma and migrated slightly faster than normal vWF (Figure 3). Normal control plasma was also treated with PNGase F, which removes the vast majority of $\mathrm{N}$-glycans on glycoproteins, resulting in a band that migrated at a much faster rate than both WT and patient vWF (Figure 3).

\subsection{FVIII secretion in STT3A and STT3B deficient cells}

FVIII level in patient plasma was too low to be analyzed by conventional biochemical methods. To analyze the impact of STT3A and STT3B on FVIII secretion, we expressed FVIII in WT, $S T T 3 A^{-/-}$or $S T T 3 B^{-/-}$HEK293 cells. ${ }^{13}$ Compared to WT cells, FVIII antigen (Figure 4A) and activity (Figure 4B) levels in conditioned media were reduced to less than $10 \%$ in $S T T 3 A^{-/-}$cells, and to between 30 and $40 \%$ in $S T T 3 B^{-/-}$cells. Intracellular levels of FVIII in $S T T 3 A^{-/-}$cells were comparable to WT cells, but lower in $S T T 3 B^{-1-}$ cells (Figure 4A).

\section{4 | DISCUSSION}

In summary, our patient with a novel homozygous variant c.1079A $>C$ (p.Tyr360Ser) in $S T T 3 A$ has many of the previously described features of STT3A-CDG including seizures, 
developmental delay, intellectual disability, microcephaly, stereotypic movements, absent speech, esotropia, and a type I CDT profile. She had onset of intractable seizures at 3 months of age (previous reports are between 4 and 15 months of age), which persist on multiple anti-epileptics at 3 years of age. It is unclear why the patient regressed in her language skills. Previous patients reported with STT3A-CDG have had absent speech, therefore it is unclear whether our patient had ever developed distinct words at baseline that would be intelligible to strangers. The p.Tyr360Ser in our patient is nestled within the transmembrane domain of STT3A as opposed to the p.Val626Ala, which is located on the luminal side of the polypeptide chain. Supplemental Figure 1, Supporting Information shows the location of the current and previously reported variants in STT3A.

In addition, our patient has chronically low FVIII, vWF, and ATIII levels, but normal FIX, FXI, Protein C, and S. She does not have pathogenic variants in $V W F, F 8$, and SERPINC1, ruling out a co-inherited von Willebrand Disease, Hemophilia A, or ATIII deficiency. Her coagulation profile is unique from the typical CDG pattern of low FIX, FXI, ATIII, Protein C, and S. ${ }^{14-16}$ Factor VIII activity was not measured in patients from the previous report, ${ }^{4}$ but partial coagulation profiles were obtained in some patients (personal correspondence with author). Case 4 had recurrent epistaxis; his APTT was normal at 34 seconds, but had been prolonged in the past (42.7-43.8 seconds). His brother (Case 3) did not have episodes of bleeding or clotting, but had prolonged APTT (47.2 seconds) and a slightly prolonged PT (14.3 seconds). Their female sibling with isolated TUSC3 deletion and no STT3A variants had a slightly prolonged PT (13.5 seconds) but normal APTT (32.9 seconds). The imbalance of coagulation factors may lead to clinical thrombosis or bleeding diathesis in some patients with CDG, particularly when undergoing surgery. ${ }^{17}$ Our patient has not had any clinically significant bleeding or thrombotic events, although she has not undergone any surgical procedures or severe metabolic stressors. The clinical features of our patient and all previously reported cases are summarized in Supplemental Table 1.

Previous mutagenesis studies showed that impeding N-glycosylation of four out of $16 \mathrm{~N}$ glycosylation sites in $V W F$ decreases or abolishes secretion of vWF. ${ }^{18}$ De-glycosylated vWF migrates faster than both mock-treated normal $v W F$ and patient $v W F$, indicating $v W F$ in our patient plasma is present in a slightly hypoglycosylated form but appears to retain most $\mathrm{N}$-glycans. This suggests reduced vWF secretion in our patient is due to the lack of at least one critical N-glycan that is $S T T 3 A$-dependent. It is possible that only mildly hypoglycosylated $\mathrm{vWF}$ is detectable in patient plasma, and additional, more severely hypoglycosylated and secretion-deficient $V W F$ variants exist in the ER of endothelial cells in vivo.

FVIII is an essential blood coagulation protein in the intrinsic pathway and normally circulates in plasma while bound to vWF, which plays an essential role in FVIII production, stabilization, and conformation. ${ }^{19,20}$ Defects in FVIII result in the X-linked bleeding disorder Hemophilia A. FVIII contains 25 consensus $\mathrm{N}$-glycosylation sites and inhibition of $\mathrm{N}$-glycosylation has been demonstrated to impair FVIII secretion. ${ }^{21} \mathrm{~N}$-glycans appear critical for FVIII folding, ${ }^{22}$ quality control, ${ }^{23}$ and ER export. ${ }^{24,25}$ Pathogenic missense variants across all domains of $F V I I I$ that create $S T T 3 A$ and $S T T 3 B$-dependent ectopic Nglycosylation sites have been demonstrated to confer a more severe phenotype of hemophilia 
A than single amino acid substitutions that do not cause ectopic N-glycosylation. ${ }^{10}$ In contrast, our patient with $S T T 3 A$-dependent hypoglycosylation and decreased secretion of FVIII has not had severe bleeding episodes similar to patients with hemophilia A due to $S T T 3 A$ and $S T T 3 B$-dependent ectopic N-glycosylation of FVIII. This is perhaps due to only mild decreases in FVIII and vWF levels and a concurrent decrease in the ATIII level, which mitigates the bleeding effects of low vWF and FVIII.

Our results indicate that STT3A deficiency leads to a severe defect in FVIII secretion and lower vWF levels of unclear clinical significance, while STT3B deficiency leads to only a mild decrease in FVIII secretion (Figure 4). This suggests that certain STT3A-dependent Nglycans required for efficient FVIII secretion are affected in our patient. Using the nGlycosylChecker program (https://github.com/MVesuviusC/nGlycosylChecker), ${ }^{10}$ we found only one hemophilia A mutation (c.779C > G, p.Ser260Cys) in the FVIII variant databases that leads to hypoglycosylation, abolishing the second $\mathrm{N}$-glycosylation sequon (N258) in the A1 domain of FVIII. This mutation reportedly caused a severe phenotype $\left({ }^{26}\right.$ suggesting that the $\mathrm{N}$-glycan at this site is important for FVIII secretion and/or function. Whether decreased FVIII secretion/activity in our patient is due to reduced occupancy of the N258 sequon or other sequons remains to be determined. The mild decrease in FVIII level in our patient likely results from the hypomorphic nature of the $S T T 3 A$ mutation (Figure 2), and is a combined effect of both decreased FVIII secretion and lower plasma VWF level.

Increased levels of hypoglycosylated pSAP species in the patient's fibroblasts indicate this novel variant in $S T T 3 A$ does exhibit a functional consequence, leading to decreased glycosylation of $S T T 3 A$-dependent substrates. This provides a mechanistic explanation for our patient's chronically low FVIII levels and activity (Figures 2-3, and 4). Our findings provide first evidence of the likely pathogenicity of the novel variant c.1079A $>\mathrm{C}$ (p.Tyr360Ser) in $S T T 3 A .{ }^{27,28}$ Patients with STT3A-CDG may have a predisposition for coagulopathy due to aberrant $\mathrm{N}$-glycosylation that may be distinct from patients with other CDGs. Although they are at risk for episodes of hemorrhage or thrombosis, their clinical phenotype is attenuated compared to patients with classic hemophilia type A due to pathogenic variants in FVIII leading to ectopic STT3A and STT3B-dependent glycosylation. The full phenotypic spectrum of STT3A-CDG and clinical impact of these coagulation defects remain to be elucidated.

\section{Supplementary Material}

Refer to Web version on PubMed Central for supplementary material.

\section{ACKNOWLEDGEMENTS}

\footnotetext{
We would like to thank Dr. Heather C. Mefford, Dr. Daniel Doherty, and Jennifer Dempsey at the University of Washington Hindbrain Malformation Research Program for their assistance with obtaining the patient's biopsy. Sequencing was provided by the University of Washington Center for Mendelian Genomics (UW CMG) and was funded by the National Human Genome Research Institute and the National Heart, Lung and Blood Institute grant 2UMIHG006493 to Drs. Debbie Nickerson, Suzanne Leal, and Michael Bamshad. We also thank Dr. Arunabha Ghosh's team for providing clinical information about their previously reported cases. We thank the family for allowing us to report this case.
} 
Funding: IC is supported by the National Institutes of Health T32GM007454 and the Shire ACMGF Medical Biochemical Genetics Award. HB was supported by T32GM007454 at the time of involvement. This work was partially supported by grants from the National Institutes of Health (R01HL094505 to BZ and R01GM43768 to RG). HHF and BGN are supported by The Rocket Fund and R01DK99551.

Funding information

National Heart, Lung, and Blood Institute, Grant/Award Number: HL094505; National Institute of Diabetes and Digestive and Kidney Diseases, Grant/Award Numbers: DK99551, DK99551; National Institute of General Medical Sciences, Grant/Award Numbers: GM007454, GM007454, R01GM43768; The Rocket Fund, Grant/ Award Numbers: $\mathrm{n} / \mathrm{a}, \mathrm{n} / \mathrm{a}$

\section{REFERENCES}

1. Cylwik B, Naklicki M, Chrostek L, Gruszewska E. Congenital disorders of glycosylation. Part I. Defects of protein N-glycosylation. Acta Biochim Pol 2013;60:151-161. [PubMed: 23730680]

2. Helenius A, Aebi M. Intracellular functions of N-linked glycans. Science 2001;291:2364-2369. 10.1126/science.291.5512.2364. [PubMed: 11269317]

3. Ruiz-Canada C, Kelleher DJ, Gilmore R. Cotranslational and post-translational N-glycosylation of polypeptides by distinct mammalian OST isoforms. Cell 2009;136:272-283. 10.1016/j.cell. 2008.11.047. [PubMed: 19167329]

4. Ghosh A, Urquhart J, Daly S, et al. Phenotypic heterogeneity in a congenital disorder of glycosylation caused by mutations in STT3A. J Child Neurol 2017;32:560-565. 10.1177/0883073817696816. [PubMed: 28424003]

5. Shrimal S, Ng BG, Losfeld M-E, et al. Mutations in STT3A and STT3B cause two congenital disorders of glycosylation. Hum Mol Genet 2013;22:4638-4645. 10.1093/hmg/ddt312. [PubMed: 23842455]

6. Krasnewich D, O'Brien K, Sparks S. Clinical features in adults with congenital disorders of glycosylation type Ia (CDG-Ia). Am J Med Genet C Semin Med Genet 2007;145C:302-306. 10.1002/ajmg.c.30143. [PubMed: 17639595]

7. Simon MT, Ng BG, Friederich MW, et al. Activation of a cryptic splice site in the mitochondrial elongation factor GFM1 causes combined OXPHOS deficiency. Mitochondrion 2017;34:84-90. 10.1016/j.mito.2017.02.004. [PubMed: 28216230]

8. Lacey JM, Bergen HR, Magera MJ, et al. Rapid determination of transferrin isoforms by immunoaffinity liquid chromatography and electrospray mass spectrometry. Clin Chem 2001;47:513-518. [PubMed: 11238305]

9. Eklund EA, Newell JW, Sun L, et al. Molecular and clinical description of the first US patients with congenital disorder of glycosylation Ig. Mol Genet Metab 2005;84:25-31. 10.1016/j.ymgme. 2004.09.014. [PubMed: 15639192]

10. Wei W, Misra S, Cannon MV, et al. Molecular mechanisms of missense mutations that generate ectopic N-glycosylation sites in coagulation factor VIII. Biochem J 2018;475:873-886. 10.1042/ BCJ20170884. [PubMed: 29444815]

11. Wei W, Zheng C, Zhu M, et al. Missense mutations near the N-glycosylation site of the A2 domain lead to various intracellular trafficking defects in coagulation factor VIII. Sci Rep 2017;7: 45033 10.1038/srep45033. [PubMed: 28327546]

12. Achouitar S, Mohamed M, Gardeitchik T, et al. Nijmegen paediatric CDG rating scale: a novel tool to assess disease progression. J Inherit Metab Dis 2011;34:923-927. 10.1007/s10545-011-9325-5. [PubMed: 21541726]

13. Cherepanova NA, Gilmore R. Mammalian cells lacking either the cotranslational or posttranslocational oligosaccharyltransferase complex display substrate-dependent defects in asparagine linked glycosylation. Sci Rep 2016;6:20946 10.1038/srep20946. [PubMed: 26864433]

14. Arnoux JB, Boddaert N, Valayannopoulos V, et al. Risk assessment of acute vascular events in congenital disorder of glycosylation type Ia. Mol Genet Metab 2008;93:444-449. 10.1016/ j.ymgme.2007.11.006. [PubMed: 18093857]

15. Fiumara A, Barone R, Buttitta P, et al. Haemostatic studies in carbohydrate-deficient glycoprotein syndrome type I. Thromb Haemost 1996;76:502-504. [PubMed: 8902985] 
16. Van Geet C, Jaeken J. A unique pattern of coagulation abnormalities in carbohydrate-deficient glycoprotein syndrome. Pediatr Res 1993; 33:540-541. 10.1203/00006450-199305000-00024. [PubMed: 8511030]

17. Linssen M, Mohamed M, Wevers RA, et al. Thrombotic complications in patients with PMM2CDG. Mol Genet Metab 2013;109: 107-111. 10.1016/j.ymgme.2013.02.006. [PubMed: 23499581]

18. McKinnon TAJ, Goode EC, Birdsey GM, et al. Specific N-linked glycosylation sites modulate synthesis and secretion of von Willebrand factor. Blood 2010;116:640-648. 10.1182/ blood-2010-02-267450. [PubMed: 20418283]

19. Federici AB. The factor VIII/von Willebrand factor complex: basic and clinical issues. Haematologica 2003;88:EREP02.

20. Weiss HJ, Sussman II, Hoyer LW. Stabilization of factor VIII in plasma by the von Willebrand factor. Studies on posttransfusion and dissociated factor VIII and in patients with von Willebrand's disease. J Clin Invest 1977;60:390-404. 10.1172/JCI108788. [PubMed: 17621]

21. Dorner AJ, Bole DG, Kaufman RJ. The relationship of N-linked glycosylation and heavy chainbinding protein association with the secretion of glycoproteins. J Cell Biol 1987;105:2665-2674. 10.1083/jcb.105.6.2665. [PubMed: 3121636]

22. Swaroop M, Moussalli M, Pipe SW, Kaufman RJ. Mutagenesis of a potential immunoglobulinbinding protein-binding site enhances secretion of coagulation factor VIII. J Biol Chem 1997;272: 24121-24124. [PubMed: 9305856]

23. Pipe SW, Morris JA, Shah J, Kaufman RJ. Differential interaction of coagulation factor VIII and factor V with protein chaperones calnexin and calreticulin. J Biol Chem 1998;273:8537-8544. [PubMed: 9525969]

24. Zheng C, Page RC, Das V, et al. Structural characterization of carbohydrate binding by LMAN1 protein provides new insight into the endoplasmic reticulum export of factors $\mathrm{V}(\mathrm{FV})$ and VIII (FVIII). J Biol Chem 2013;288:20499-20509. 10.1074/jbc.M113.461434. [PubMed: 23709226]

25. Zhu M, Zheng C, Wei W, et al. Analysis of MCFD2- and LMAN1-deficient mice demonstrates distinct functions in vivo. Blood Adv 2018;2:1014-1021. 10.1182/bloodadvances.2018018317. [PubMed: 29735583]

26. Vinciguerra C, Zawadzki C, Dargaud Y, et al. Characterisation of 96 mutations in 128 unrelated severe haemophilia A patients from France. Description of 62 novel mutations. Thromb Haemost 2006;95:593-599. [PubMed: 16601827]

27. Nykamp K, Anderson M, Powers M, et al. Sherloc: a comprehensive refinement of the ACMGAMP variant classification criteria. Genet Med 2017;19:1105-1117. 10.1038/gim.2017.37. [PubMed: 28492532]

28. Richards S, Aziz N, Bale S, et al. Standards and Guidelines for the Interpretation of Sequence Variants: a Joint Consensus Recommendation of the American College of Medical Genetics and Genomics and the Association for Molecular Pathology. Genet Med; 2015;17(5):405-424. 10.1038/gim.2015.30. [PubMed: 25741868] 

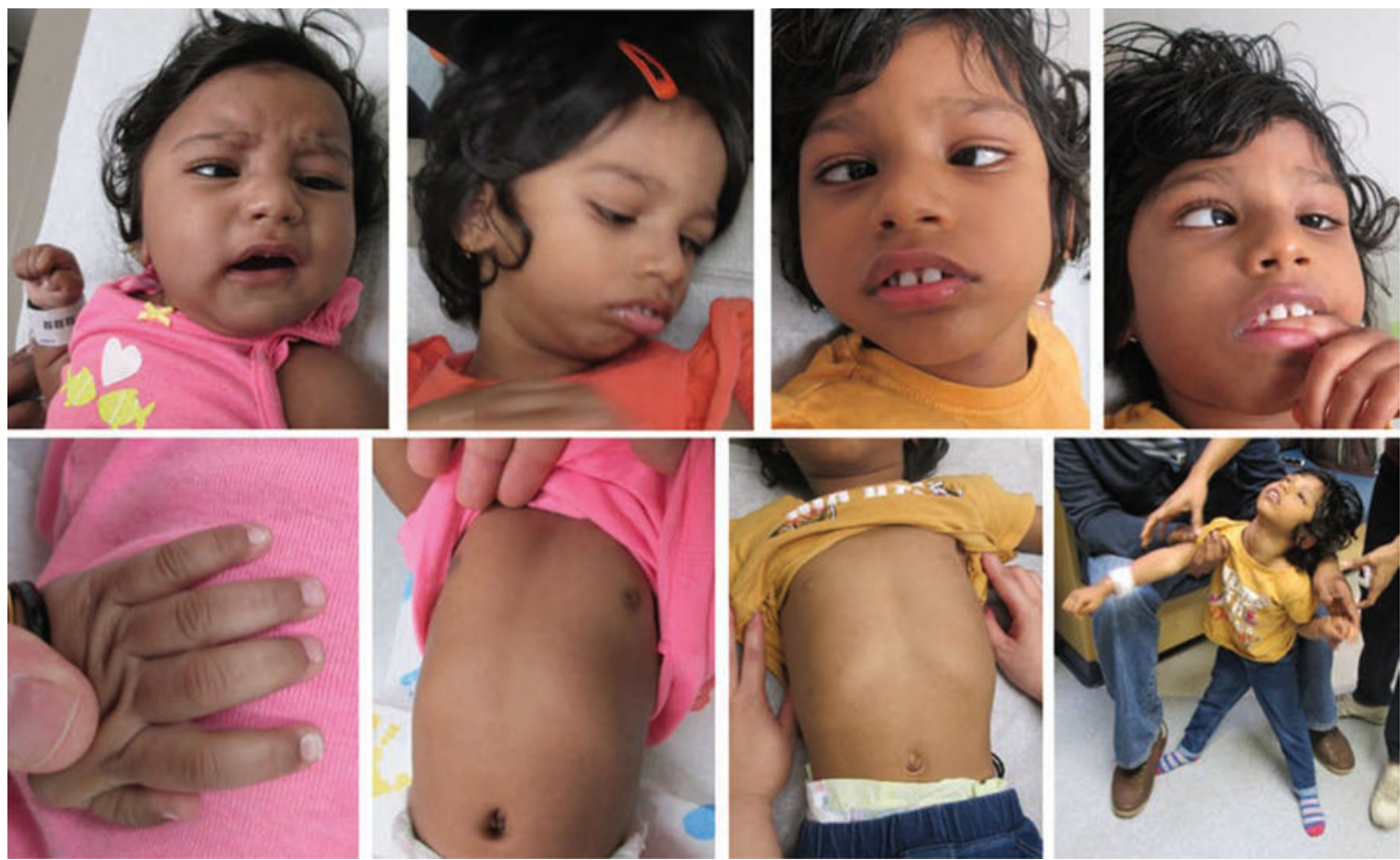

FIGURE 1.

Patient photographs at 3 months (upper and lower left), 3 years (upper center left), and 3.5 years of age (upper center right and upper right) showing esotropia and ptosis. Inverted nipples were noted at 3 months of age (lower center left), which resolved by 3 years (lower center right). Patient is able to weight bear at 3.5 years with support (lower right) 
(A)

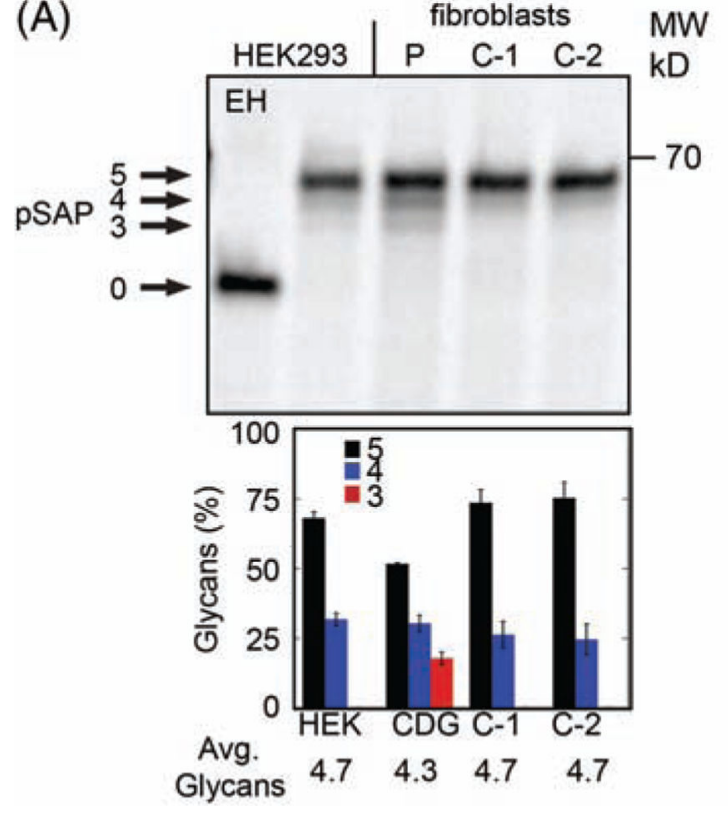

(B)

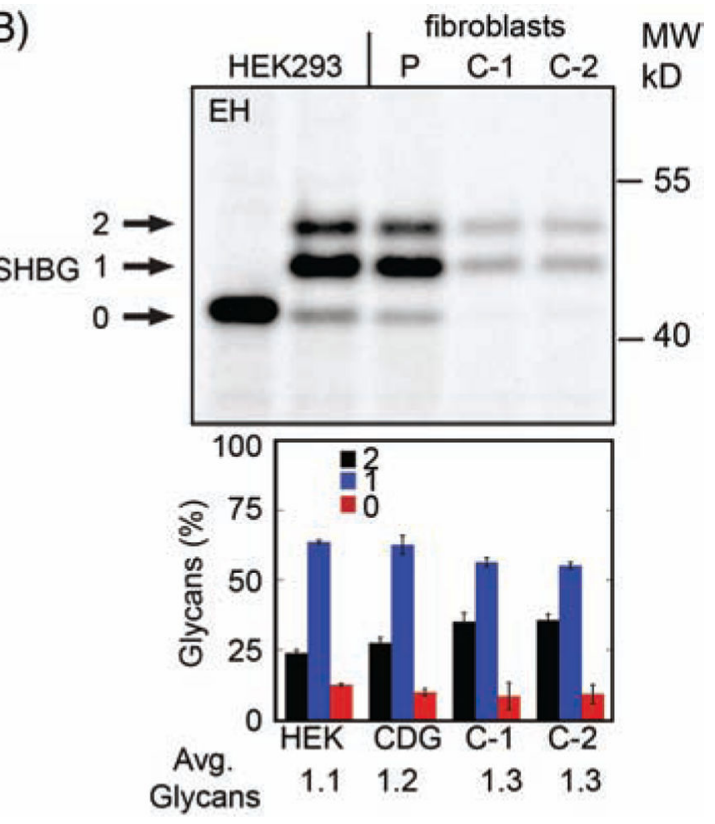

\section{FIGURE 2.}

Panel A - increased amount of pSAP chains with three glycans in skin fibroblasts of the patient compared to controls by pulse labeling. Panel B - no reduction in glycosylation of sex hormone binding globulin (SHBG) in skin fibroblasts by pulse labelling. Labeled arrows on the left side of images designate the number of glycans. The quantified percentage of chains with the indicated number of glvcans (bar graph) was used to determine the average number of glycans per protein chain. The values shown are the average of two determinations, with error bars designating individual data points. Normal control bands are lighter due to decreased transfection efficiency, but this does not impact the average number of glycans per chain. pSAP: Prosaposin; P: Patient fibroblast line; C-1 and C-2: Control fibroblast lines; MW, molecular weight; kD, kilo Dalton; Avg, average 


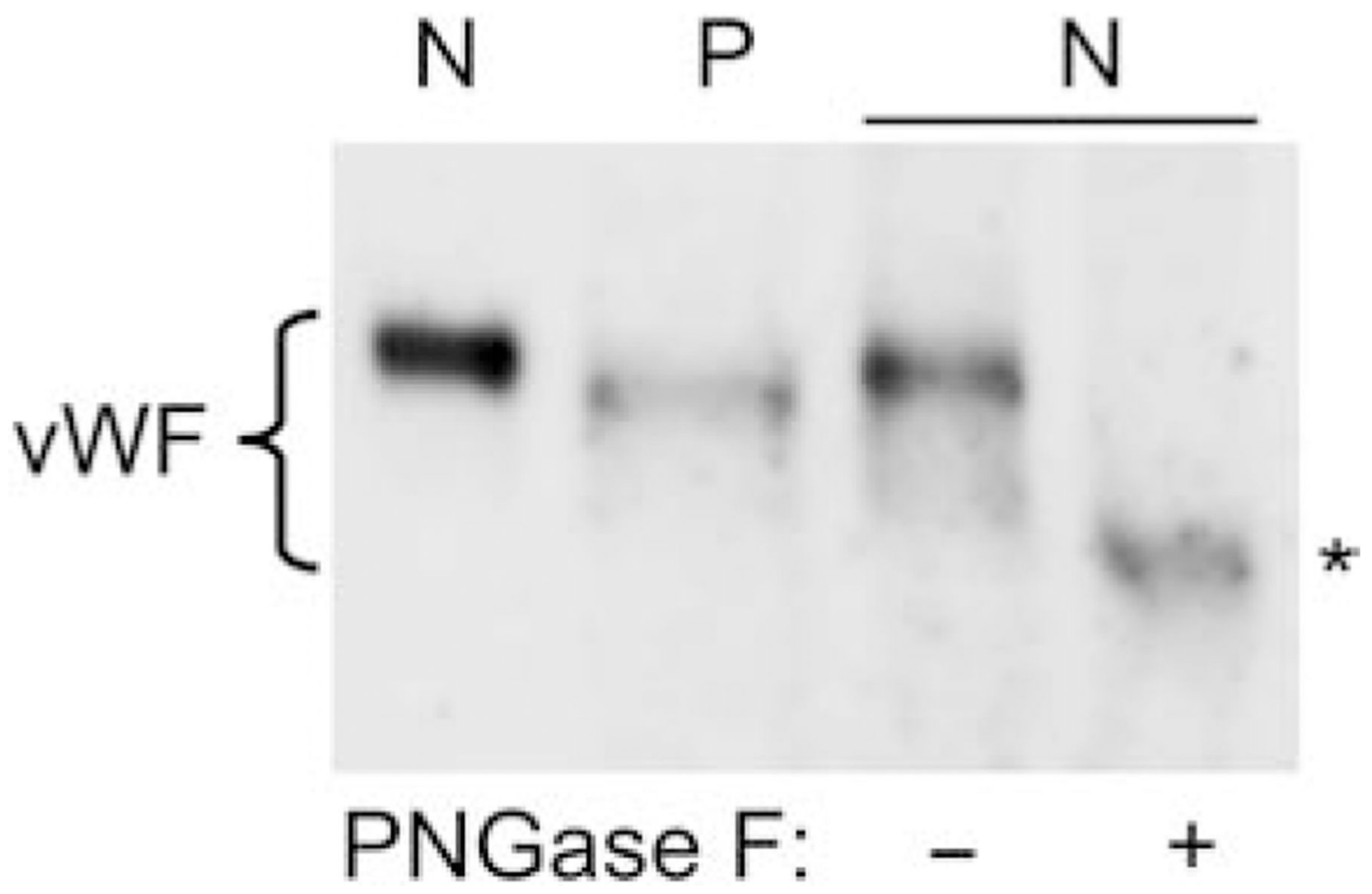

FIGURE 3.

Western blot analysis of plasma VWF. Left two lanes: Plasma samples from normal control $(0.5 \mu \mathrm{L})$ and patient $(2.5 \mu \mathrm{L})$. Right two lanes: normal control plasma samples $(0.9 \mu \mathrm{L}$ each) were mock treated (-) or treated (+) with PNGase F. Asterisk indicates the VWF band with $\mathrm{N}$-glycans removed. $\mathrm{N}$, normal control; $\mathrm{P}$, patient; VWF, von Willebrand factor 
(A)

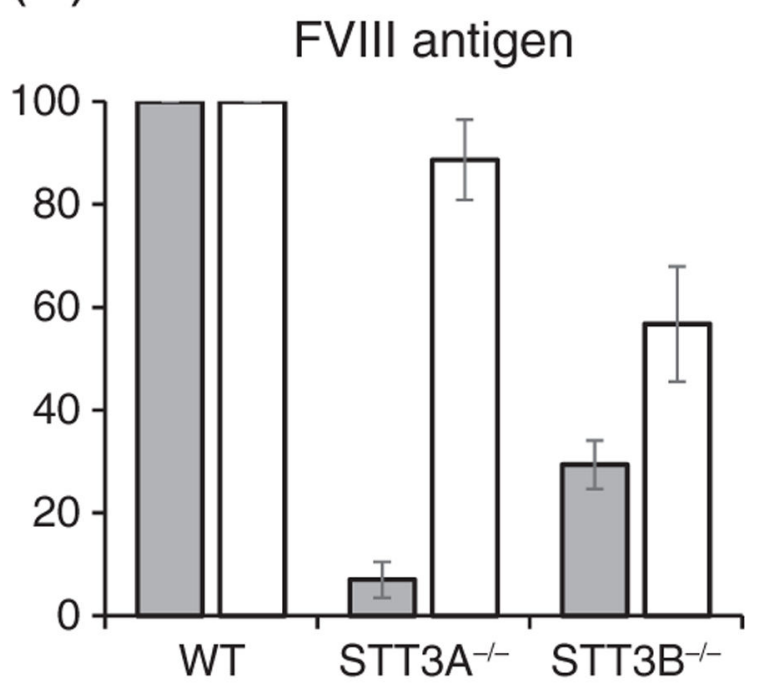

(B)

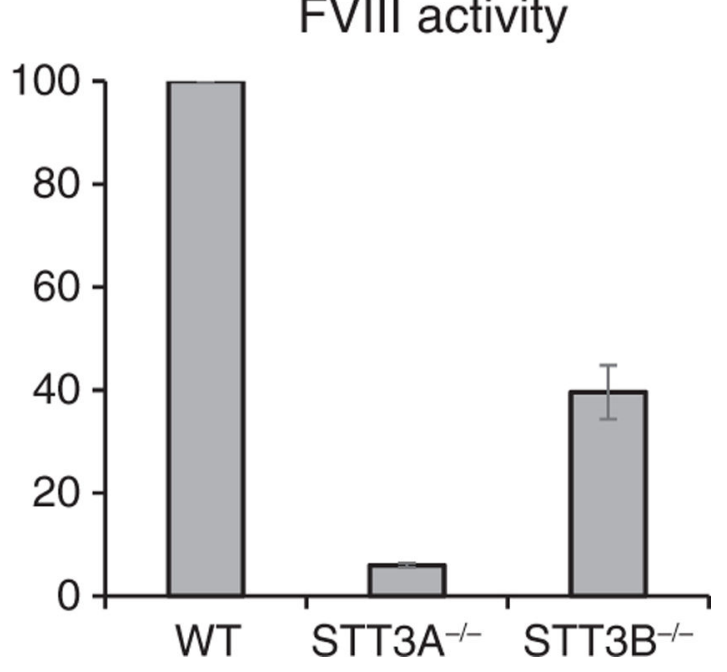

FIGURE 4.

FVIII secretion in STT3A and STT3B deficient cells. pMT2-FVIII plasmid was transiently transfected into WT, $S T T 3 A^{-/-}$and $S T T 3 B^{-/-}$HEK293 cells. Forty-eight hours post transfection, conditioned media, and cell lysates were collected and analyzed. A, FVIII antigen levels in conditioned media (grey bars) and in cell lysates (white bars). B, FVIII activities in conditioned media. FVIII antigen and activity levels in $S T T 3 A^{-/-}$and $S T T 3 B^{-1-}$ HEK293 cells were plotted as percentages of WT levels. Error bars represent one SD of three experiments 